\title{
Adaptive feed-forward controller of piezoelectric actuator for micro/nano-positioning
}

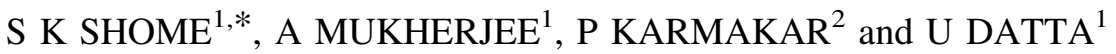 \\ ${ }^{1}$ Academy of Scientific and Innovative Research (AcSIR), Central Mechanical Engineering Research Institute, \\ Council of Scientific and Industrial Research Campus, Durgapur 713209, India \\ ${ }^{2}$ Central Mechanical Engineering Research Institute, Council of Scientific and Industrial Research, Durgapur, \\ India \\ e-mail: saikatkumarshome@gmail.com
}

MS received 26 May 2017; revised 16 March 2018; accepted 10 April 2018; published online 14 August 2018

\begin{abstract}
In this paper, design and implementation of an adaptive feed-forward controller for micro/nanopositioning control of piezoelectric actuator (PEA) is described. Discrete-time Dahl hysteresis-based mathematical model of the PEA is developed and the values of the model parameters are estimated through an autoregressive with exogenous terms (ARX) model identification technique using experimental input-output data. A recursive least-square estimator (RLSE)-based adaptive feed-forward (FF) controller is proposed, which takes into account the parameter uncertainty. The FF controller has also been implemented in a DsPIC30F4011 microcontroller. The established PEA model and the controller are validated by simulation and experimental results including parameter variation.
\end{abstract}

Keywords. Adaptive control; piezoelectric actuator; micro/nano-positioning; RLSE.

\section{Introduction}

With rapid development in engineering and technology, everything goes for miniaturization, which necessitates ultra-precise positioning systems in industrial applications. Quite a few system applications call for positioning accuracy down to nanometre resolution. Recently, the domain of precision positioning has been dominated by a class of actuators called piezoelectric actuators (PEAs) and have emanated to be the most favoured class of actuators in this field. The eminence of PEA can be attributed to some of its inherent advantages, e.g., large operating bandwidth, generate large mechanical force per unit mass with low power consumption, small thermal expansion during actuation, fast response and high resolution.

Typically, micro/nano-manipulation with dynamically interactive application needs actuators that can accurately perform reference tracking as well as yield robust force control. Nowadays, PEA has found widespread commercial applications in the field of optics, photonics, measuring technology, nano-metrology, micro-lithography, scanning microscopy, etc. [1-4]. Precision motion control using piezo-actuators has been reported to be a promising

*For correspondence approach and numerous research works have reported improving its efficiency in recent times [5-13]. Al-Wahab et al [14] have presented a methodology for the appropriate selection and creation of mechatronic handling devices, which can be capable of accomplishing certain micro- and/ or nano-operation task using piezo-actuators.

Piezo-actuators also find considerable usage in highprecision dual-stage servo systems which has been reported in [15].

Though PEA is a promising actuator, these objectives are severely hampered due to some inherent nonlinearity like hysteresis, creep and vibration, the effects of which get more pronounced at smaller scales. However, they can be minimized with proper modelling and control. Out of these, the primary nonlinearity that adversely effects precision positioning is hysteresis. Hysteresis is also known for introducing limit cycles and chattering in addition to introducing severe positioning inaccuracies. Besides, the variation of system dynamics with environmental and loading condition also needs to be considered during control of a piezoelectric-material-actuated platform. Furthermore, the presence of creep in PEA causes a change in displacement with time without any accompanying change in the control voltage. This degrades the positioning accuracy of the system. This necessitates the requirement of a controller that can change its behaviour in response to the modification in dynamics of the processes and the disturbances acting on the system along with proper system 
modelling. Accordingly, an adaptive feed-forward (FF) control system can be utilized. The effects of hysteresis of piezo-actuators have been modelled and compensated by Viswamurthy and Ganguli [16] and Viswamurthy et al [17] to reduce trailing edge flap vibration in helicopters. Liaw and Shirinzadeh [18] have presented a robust adaptive constrained motion tracking control methodology for piezoactuated flexure-based micro/nano-manipulation mechanisms. This unique control approach is established for the tracking of desired motion trajectories in a constrained environment exhibiting some degree of uncertainty in stiffness. In order to improve the high-resolution positioning performances of the microgripper, its actuators are modelled and a control law for both the position and the manipulation force is synthesized in [19]. In [20], a study of cascaded feedback and FF controller is presented to compensate hysteresis, creep and vibration effects of a piezoscanner for high-speed positioning in AFM.

Constant gains in traditional feedback may increase the oscillations in the control signal and induce the excitation of high-frequency unmodelled dynamics of the system, which are undesirable phenomena [21]. To overcome this drawback, variable gains with adaptive rules have been reported in literature and found to be effective in the domain of piezo-actuation. Adaptive control is a dynamic and pivotal field of research in industrial applications. Over the past decade, importance of several self-adaptation-related application areas and technologies has increased [22-26]. It has also been applied in PEA systems by a number of researchers. In [27], an improved sliding mode control with PID-type sliding surface and adaptive gains is proposed for the motion tracking of PEA, where the controller requires only the online estimation of perturbation and control gains without acquiring the knowledge of bounds on system uncertainties. An adaptive proportionalintegral (PI)-based sliding mode control design is proposed in [28] for nano-positioning of PEA, where the hysteresis and model imperfections are treated as uncertainties and addressed by sliding mode control. In [29], an adaptive approximator-based back-stepping control approach is proposed for trajectory tracking of the single-axis PEAdriven stages, which can provide an improvement in transient performance and tracking errors, and robustness to the load disturbance.

A new adaptive controller structure is introduced in [30], which is capable of achieving a linear parameterization and a linear error model in the presence of a hysteresis nonlinearity. A robust adaptive law is used to update the controller parameters and hysteresis inverse parameters, which ensures the global boundedness of the closed-loop signals for a wide class of hysteresis models. An adaptive sliding mode control method is proposed in [31] for eliminating hysteresis effect and achieving highly precise output displacement of PEAs. Paper [32] presents an adaptive model predictive control technique for reducing hysteresis in PEAs based on autoregressive exogenous (ARX) model. It is inferred that an adaptive controller with adjustable specification and mechanism for adjusting parameters is preferred for piezo-actuation where the parameters can be modified to compensate for the shift in dynamics of the system and the disturbances acting on the plant.

The fulfilment of any control paradigm depends on the choice of a suitable mathematical modelling of the physical phenomenon. Quite a few framework models have been studied that represent system performance to an appropriate degree. These frameworks provide a generic intuition into the behaviour of PEA and also lay mathematical formulations for advanced control system design and realization. Various methods have been developed for modelling of the nonlinearities in PEA such as the Maxwell Slip Model, Preisach Model, Bouc-Wen Model and Dahl model [33-38]; however, the latter two are the most commonly used. In this research, plant dynamics have been captured using a second-order Dahl model, which has been cited to be better than the second-order BoucWen model in depicting asymmetric hysteresis loops [38]; moreover, it has less number of parameters and it is easy to construct the inverse model. Though the Dahl model has been reported to be a promising approach, majority of works have been carried out on continuous-time base. Discrete-time Dahl hysteresis model is more preferable as control schemes are usually deployed for sampled data systems.

In this paper a new recursive least-square estimator (RLSE)-based adaptive discrete-time FF controller is developed and implemented on DsPIC30F4011 microcontroller platform. The dynamical model of PEA actuator is represented by a mass-spring-damper system with hysteresis force that is designed using a Dahl model [38] in continuous-time domain. The PEA model along with Dahl hysteresis model has been converted to discrete-time domain. The system parameters are estimated using the ARX model identification method with the help of several experimental input-output data. However, since the system parameters change with time, an uncertainty factor in the parameters has been incorporated in the system model. The values of the parameters are updated in each time step by RLSE-based adaptive FF control. The control algorithm is implemented on a DsPIC30F4011 microcontroller. The developed discrete-time PEA model has been validated by comparing the simulation and experimental results using the same sinusoidal signal of multiple amplitudes and frequencies. The adaptive FF controller is also validated with simulation and experimental results with different input signals. The rest of this paper is organized as follows. In section 2, the mathematical modelling of PEA in continuous- and discrete-time domain is described. Section 3 focuses on model identification. Section 4 describes the proposed RLSE-based adaptive FF controller that generates the control voltage. Results are given in section 5, and conclusions are drawn in section 6. 


\section{Mathematical model of PEA}

\subsection{Continuous-time PEA model}

In continuous-time domain, the dynamic model of PEA can be expressed in the form of a mass-spring-damper system with hysteresis force. The same is modelled using the Dahl hysteresis model [38], by the equation

$$
M \ddot{x}+D \dot{x}+K x=T u-F_{h}
$$

where $M$ is the mass of the system, $D$ is damping co-efficient, $K$ is stiffness, $T$ is piezoelectric coefficient, $u$ is input voltage and $F_{h}$ is the hysteresis effect of the system in terms of force. The Dahl hysteresis model can be represented by the following equations:

$$
\begin{gathered}
\dot{V}=A_{h} V \dot{x}+B_{h} u_{p} \dot{x} \\
F_{h}=C_{h} V
\end{gathered}
$$

where the variable $x$ denotes the displacement, $V=\left[\begin{array}{ll}v_{1} & v_{2}\end{array}\right]^{\mathrm{T}}$ is an intermediate state vector for the second-order model, $u_{p}$ is taken as constant and the matrices can be written as follows:

$$
\begin{aligned}
A_{h} & =\left[\begin{array}{cc}
0 & 1 \\
-a_{2} & -\operatorname{sign}(\dot{x}) a_{1}
\end{array}\right], B_{h}=\left[\begin{array}{l}
0 \\
1
\end{array}\right] \text { and } \\
C_{h} & =\left[\begin{array}{ll}
b_{1} & \operatorname{sign}(\dot{x}) b_{0}
\end{array}\right]
\end{aligned}
$$

In Eq. (3), $a_{1}, a_{2}, b_{0}$ and $b_{1}$ are hysteresis parameters and can be found from the following equations:

$$
\begin{gathered}
a_{1}=\frac{2}{T_{d}} \ln \frac{P_{n-1}-P_{s}}{P_{n}-P_{s}} \\
a_{2}=\frac{4 \pi^{2}}{T_{d}^{2}}+\frac{a_{1}^{2}}{4} \\
b_{1}=G_{d c} a_{2} \\
b_{0}=S_{0}
\end{gathered}
$$

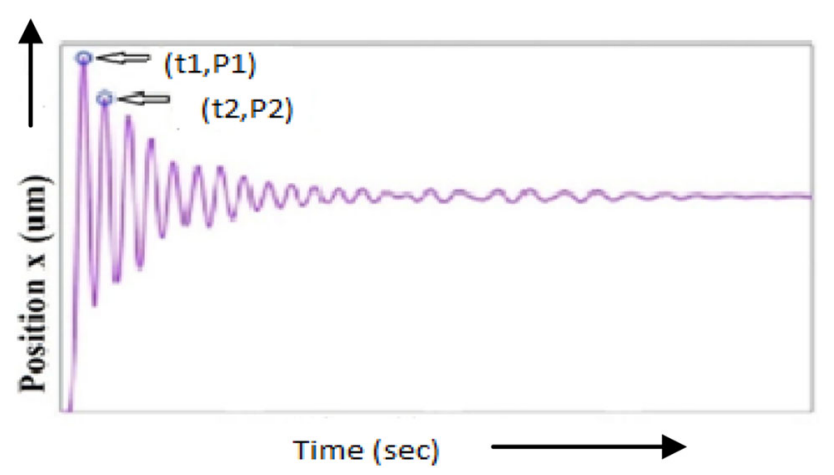

Figure 1. Open-loop time domain step response for a piezoelectric actuator. where $T_{d}$ is the time period of the damped oscillations of the system, which is equal to $\left(t_{2}-t_{1}\right)$ from figure $1 . P_{n}$ is the overshoot of the $n^{\text {th }}$ peak and $P_{s}$ is the steady-state response. $G_{d c}$ represents the initial gain and $S_{0}$ is the initial slope of the response in FFT when the system is given a step signal.

\subsection{Discrete-time PEA model}

For a digital control system, discrete-time system is more suitable than continuous-time system. Hence, the continuous PEA model is converted to a discrete-time domain. The differential equations (1)-(3) are converted into difference equations.

If the sampling time is $h$, then at the $k^{\text {th }}$ time instant

$$
\begin{aligned}
& \dot{x}(t)=\frac{x(k+1)-x(k)}{h} \\
& \ddot{x}(t)=\frac{\dot{x}(k+1)-\dot{x}(k)}{h}
\end{aligned}
$$

Using (8), we get

$$
\ddot{x}(\mathrm{t})=\frac{x(k)-2 x(k-1)+x(k-2)}{h^{2}} .
$$

Now, replacing the value of $\dot{x}(t)$ and $\ddot{x}(t)$ in (1), the PEA discrete-time model can be given as

$$
x(k)=A x(k-1)+B x(k-2)+C u(k)+D F_{h}(k)
$$

where $A=\frac{\frac{2 M}{h^{2}}+\frac{D}{h}}{\frac{M}{h^{2}}+\frac{D}{h}+k}, B=-\frac{\frac{M}{h^{2}}}{\frac{M}{h^{2}}+\frac{D}{h}+k}, C=\frac{T}{\frac{M}{h^{2}}+\frac{D}{h}+k}, D=-\frac{1}{\frac{M}{h^{2}+\frac{D}{h}+k}}$.

Now, the Dahl hysteresis force in continuous time can be expressed in discrete-time domain by replacing $\dot{V}(t)=\frac{V(k+1)-V(k)}{h}, V(k)=\left[\begin{array}{l}v_{1}(k) \\ v_{2}(k)\end{array}\right]$ and $\dot{x}(t)=\frac{x(k+1)-x(k)}{h}$ in (2a), (2b) and (2b), we get

$$
v_{1}(k)=v_{1}(k-1)+v_{2}(k)\{x(k)-x(k-1)\},
$$

$v_{2}(\mathrm{k})=$

$$
\frac{v_{2}(k-1)+u_{p}\{x(k)-x(k-1)\}-a_{2} v_{2}(k)\{x(k)-x(k-1)\}}{1+a_{1}\{x(k)-x(k-1)\} \operatorname{sgn}\left\{\frac{x(k)-x(k-1)}{h}\right\}},
$$

$$
F_{h}(k)=v_{1}(k) b_{1} \operatorname{sgn}\left\{\frac{x(k)-x(k-1)}{h}\right\} b_{0} v_{2}(k) .
$$

Taking Z-transform of (11), the model of the system in Zdomain is obtained as

$$
X(Z)=\frac{Z^{2}\left\{C U(Z)+D F_{h}(Z)\right\}}{Z^{2}-A Z-B} .
$$




\section{Model identification}

The PEA model can be assumed to be a second-order discrete-time transfer function in cascade with the Dahl-based hysteresis model. The parameters of the transfer function (figure 2a) $A-D$ are evaluated with the help of several experimental input-output data using it as a black box model. The regeneration model ARX is used to identify the values.

$$
\begin{array}{r}
F\left(q^{-1}\right) y(k)=q^{-d} H\left(q^{-1}\right) u(k)+\varepsilon(k) \\
F\left(q^{-1}\right)=1+f_{1} q^{-1}+\ldots f_{n a} q^{-n a} \\
H\left(q^{-1}\right)=h_{0}+h_{1} q^{-1}+\ldots h_{n b} q^{-n b}
\end{array}
$$

where $q$ is the delay operator, $y$ is the output vector, $u$ is the input vector and $\varepsilon$ is the error between model output and actual output. Using the ARX model, the regressing model can be defined as

$$
y(k)=\varphi^{\mathrm{T}} \theta+e(k) .
$$

Here, the vector

$$
\begin{gathered}
\varphi^{\mathrm{T}}=[-y(k-1),-y(k-2), \ldots-y(k-n), \\
u(k-d), u(k-d-1), \ldots u(k-d-m)]
\end{gathered}
$$

and unknown parameters

$$
\theta^{T}=\left[f_{1}, f_{2}, \ldots, f_{n}, h_{1}, h_{2}, \ldots, h_{n}\right] .
$$

The least-mean-square cost function is

$$
J(\hat{\theta})=\sum_{k=1}^{N}\left[y(k)-\varphi^{\mathrm{T}}(k) \hat{\theta}\right]^{2} .
$$

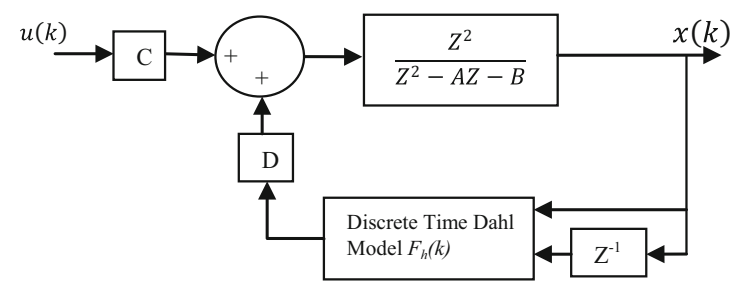

(a)

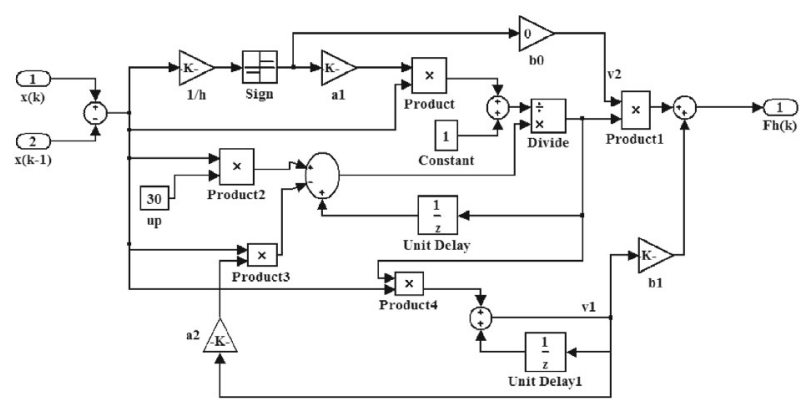

(b)

Figure 2. (a) Block diagram of piezoelectric actuator. (b) Discrete-time Dahl model.
The $\varphi^{\mathrm{T}}(k) \hat{\theta}$ is the predicted output and $y(k)$ is the real output. The derivative $\frac{\partial J(\hat{\theta})}{\partial \hat{\theta}}$ is set to zero to minimize the cost function. Therefore

$$
\begin{aligned}
\frac{\partial J(\hat{\theta})}{\partial \hat{\theta}} & =-2 \sum_{k=1}^{N} \varphi(k)\left[y(k)-\varphi^{\mathrm{T}}(k) \hat{\theta}\right] \\
& =0 \\
& =>\sum_{k=1}^{N} \varphi(k) y(k)=\left[\sum_{k=1}^{N} \varphi(k) \varphi^{\mathrm{T}}(k)\right] \hat{\theta} .
\end{aligned}
$$

Finally, the unknown parameters can be estimated as

$$
\hat{\theta}_{N}=\left[\sum_{k=1}^{N} \varphi(k) \varphi^{\mathrm{T}}(k)\right]^{-1} \sum_{k=1}^{N} \varphi(k) \mathrm{y}(k) .
$$

The least-square estimate in matrix notation is

$$
\hat{\theta}_{N}=\left[\Phi^{T} \Phi\right]^{-1} \Phi^{T} Y
$$

where

$$
\sum_{k=1}^{N} \varphi(k) \varphi^{\mathrm{T}}(k)=[\varphi(1) \ldots \varphi(N)]\left[\begin{array}{c}
\varphi^{\mathrm{T}}(1) \\
\vdots \\
\varphi^{\mathrm{T}}(N)
\end{array}\right]=\Phi^{\mathrm{T}} \Phi
$$

and

$$
\sum_{k=1}^{N} \varphi(k) y(k)=[\varphi(1) \ldots \varphi(N)]\left[\begin{array}{c}
y(1) \\
\vdots \\
y(N)
\end{array}\right]=\Phi^{\mathrm{T}} Y .
$$

Here, $\Phi$ is a full rank matrix; thus $\Phi^{\mathrm{T}} \Phi$ is invertible and least squares estimate exists.

The initial values of the parameters $A-D$ of (11) can be evaluated with the help of (18), where

$$
\varphi^{T}=\left[x(k-1), x(k-2), u(k), F_{h}(k)\right]
$$

and

$$
\theta^{\mathrm{T}}=[A, B, C, D] .
$$

Input voltage at different frequencies and amplitudes are given to the actual PEA (P-841.20) and outputs of the system are recorded. The same inputs are given to the simulation model to determine the validity of the PEA model.

\section{RLSE-based adaptive FF controller}

The FF controller is designed to generate required control voltage to travel the desired distance by the PEA. The FF control voltage can be generated with the help of the mathematical model of the PEA. The initial values of the 
parameters $(A-D)$ are estimated using the method as discussed earlier. Values of these parameters change with time; hence, uncertainty $\nabla A, \nabla B, \nabla C$ and $\nabla D$ is considered for the parameters $A, B, C$ and $D$, respectively. The PEA model (11) considering the parameter uncertainty can be represented as

$$
\begin{aligned}
x(k)= & (A+\nabla A) x(k-1)+(B+\nabla B) x(k-2) \\
& +(C+\nabla C) u(k)+(D+\nabla D) F_{h}(k) .
\end{aligned}
$$

The control voltage $u_{F F}$ is expressed as follows:

$$
u_{F F}(k)=A_{1} x(k)+B_{1} x(k-1)+C_{1} x(k-2)+D_{1} F_{h}(k) \text {. }
$$

From (22)

$$
\begin{aligned}
& A_{1}=\frac{1}{(C+\nabla C)}, B_{1}=-\frac{(A+\nabla A)}{(C+\nabla C)}, \\
& C_{1}=-\frac{(B+\nabla B)}{(C+\nabla C)}, D_{1}=-\frac{(D+\nabla D)}{(C+\nabla C)}
\end{aligned}
$$

The recursive-least-square-based method is adopted to estimate and update the unknown parameters $A_{1}, B_{1}, C_{1}$ and $D_{1}$ of the system in a dynamic manner. The RLS method is the extended type of least-mean-square algorithm and it imposes adaptability in the system.

Let us assume

$$
\begin{gathered}
P^{-1}(k)=\sum_{k=1}^{N} \varphi(k) \varphi^{\mathrm{T}}(k), \\
B(k)=\sum_{k=1}^{N} \varphi(k) y(k) .
\end{gathered}
$$

Therefore (18) can be written as

$$
\hat{\theta}_{k}=P(k) B(k)
$$

where $P(k)$ and $B(k)$ can be written in time-dependent form as

$$
\begin{gathered}
P^{-1}(k)=P^{-1}(k-1)+\varphi(k) \varphi^{\mathrm{T}}(k), \\
B(\mathrm{k})=B(k-1)+\varphi(k) y(k) .
\end{gathered}
$$

Replacing (28) in (26)

$$
\begin{array}{r}
P^{-1}(k) \hat{\theta}_{k}=B(k-1)+\varphi(k) y(k), \\
P^{-1}(k) \hat{\theta}_{k}=P^{-1}(k-1) \hat{\theta}_{k-1}+\varphi(k) y(k) .
\end{array}
$$

As from (26), we can write $\hat{\Theta}_{k-1}=P(k-1) B(k-1)$. Therefore $B(k-1)=P^{-1}(k-1) \hat{\theta}_{k-1}$.

Replacing (27) in (29)

$$
\begin{array}{r}
P^{-1}(k) \hat{\theta}_{k}=\left\{P^{-1}(k)-\varphi(k) \varphi^{\mathrm{T}}(k)\right\} \hat{\theta}_{k-1}+\varphi(k) y(k), \\
\hat{\theta}_{k}=\hat{\theta}_{k-1}+P(k) \varphi(k)\left[y(k)-\varphi^{\mathrm{T}}(k) \hat{\theta}(k-1)\right] .
\end{array}
$$

The matrix inverse lemma $(A+B C D)^{-1}=A^{-1}$ $A^{-1} B\left(C^{-1}+D A^{-1} B\right) D A^{-1}$ is used to derive a recursive equation for $P(k)$ rather than $P^{-1}(k)$ as in (31). Utilizing this lemma, we get the following equations for update of unknown parameters:

$$
\begin{gathered}
P(k)=P(k-1)-\frac{P(k-1) \varphi(k) \varphi^{\mathrm{T}}(k) P(k-1)}{1+\varphi^{\mathrm{T}}(k) P(k-1) \varphi(k)}, \\
\hat{\theta}_{k}=\hat{\theta}_{k-1} \\
\quad+\frac{P(k-1) \varphi(k)}{1+\varphi^{\mathrm{T}}(k) \mathrm{P}(k-1) \varphi(k)}\left[y(k)-\varphi^{\mathrm{T}}(k) \hat{\theta}(k-1)\right] .
\end{gathered}
$$

The parameters $A_{1}-D_{1}$ are updated at each time step using RLS algorithm [39-41] (31)-(32), where $\hat{\theta}_{k}^{\mathrm{T}}=\left[A_{1}, B_{1}, C_{1}, D_{1}\right]$ and $\varphi^{\mathrm{T}}(k)=\left[x(k), x(k-1), x(k-2), F_{h}(k)\right]$. The block diagram of the simulation model of RLS-based FF controller of the PEA is shown in figure 3.

\section{Results and discussion}

The model parameters $(A-D)$ of the discrete-time PEA are evaluated through model identification with the help of several experimental data, i.e., input (voltage)-output (position) data of the PEA, P841.20. The specification of the actuator is given in table 1 . The displacement of the PEA is measured by the attached Strain Gauge Sensor (SGS) with the actuator, which is displayed on the display module of E-501.00. The relation between the output voltage of the sensor and the position of the PEA is developed using several experimental data. A mathematical equation is developed from these data to find the position of the actuator from the SGS sensor voltage. The initial parameters of the discrete-time PEA are estimated by model identification using experimental input-output data as discussed in section 3; the hysteresis parameters are calculated by the method as described in section 2 . The initial values of the parameters are given in table 2 .

The output position plots of the actual PEA (P841.20) and the simulation model using the same input signals with

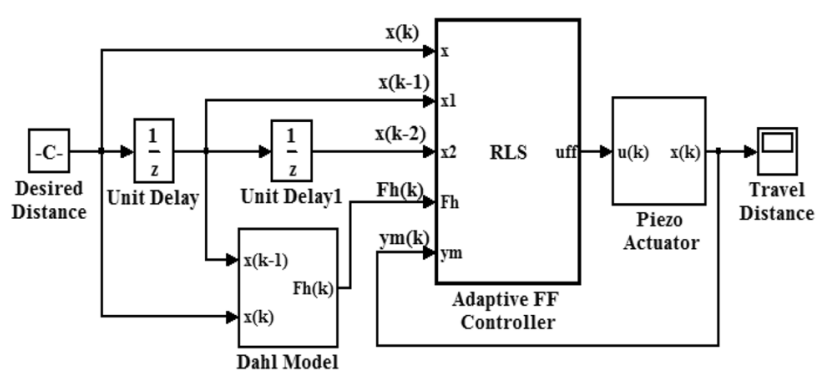

Figure 3. RLS-based FF controller. 
different amplitudes and frequencies are shown in figures 4 and 5. The sinusoidal input voltages of amplitude 10 and $50 \mathrm{~V}$ with frequency 1 and $10 \mathrm{~Hz}$ are used as an input of the PEA shown in figures $4 \mathrm{a}, 4 \mathrm{c}, 5 \mathrm{a}$ and $5 \mathrm{c}$, respectively. The output position of actual experimental data and the output from the simulation model using the same input signal are shown in figures $4 \mathrm{~b}, 4 \mathrm{~d}, 5 \mathrm{~b}$ and $5 \mathrm{~d}$. The initial parameters are updated using (31) and (32). The RLSEbased adaptive FF controller is first simulated using

Table 1. Specifications of the piezo-actuator.

\begin{tabular}{lc}
\hline Open-loop travel, at 0-100 V & $30 \mu \mathrm{m}$ \\
Closed-loop travel & $30 \mu \mathrm{m}$ \\
Integrated feedback sensor & $\mathrm{SGS}$ \\
Resolution, closed-loop/open-loop & $0.6 / 0.3 \mathrm{~nm}$ \\
Static large-signal stiffness & $27 \mathrm{~N} / \mu \mathrm{m}$ \\
Electrical capacitance & $3.0 \mu \mathrm{F}$ \\
Push force capacity & $1000 \mathrm{~N}$ \\
Length & $50 \mathrm{~mm}$
\end{tabular}

Table 2. Values of model parameters.

\begin{tabular}{lc}
\hline Parameter & Value \\
\hline$A$ & -0.43423 \\
$B$ & -0.40844 \\
$C$ & $4.3873 \times 10^{-2}$ \\
$D$ & $-4.5833 \times 10^{-5}$ \\
$a_{1}$ & 122 \\
$a_{2}$ & $1.1775 \times 10^{6}$ \\
$b_{0}$ & 0 \\
$b_{1}$ & $1.849 \times 10^{6}$ \\
\hline
\end{tabular}
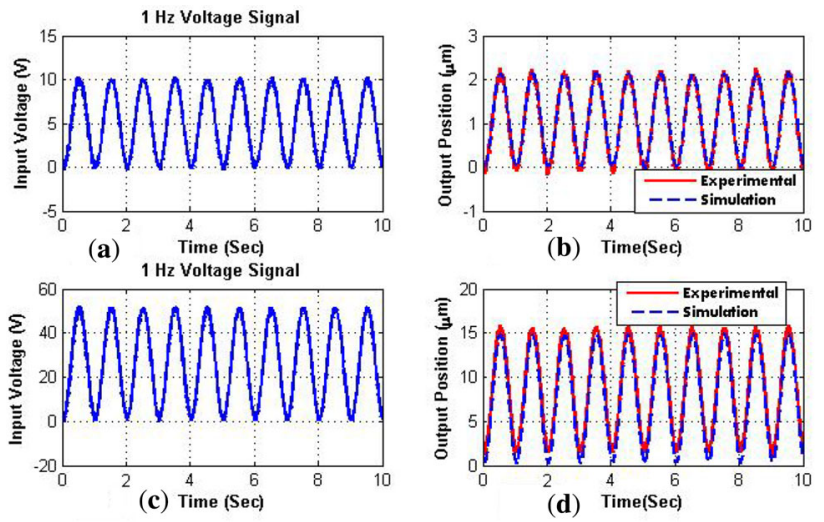

Figure 4. (a) $10 \mathrm{~V}, 1 \mathrm{~Hz}$ input voltage of PEA, (b) output position plot of experimental data and simulation model using $10 \mathrm{~V}, 1 \mathrm{~Hz}$ input voltage, (c) $50 \mathrm{~V}, 1 \mathrm{~Hz}$ input voltage of PEA and (d) output position plot of experimental data and simulation model using $50 \mathrm{~V}, 1 \mathrm{~Hz}$ input voltage.
MATLAB-SIMULINK. The block diagram is shown in figure 3. A variation of approximately $\pm 0.5 \%$ in initial values of the parameters $A-D$ is considered, i.e., the uncertainties are $\nabla A= \pm 0.005 A, \nabla B= \pm 0.005 B, \nabla C=$ $\pm 0.005 C$ and $\nabla D= \pm 0.005 D$. The desired sinusoidal trajectory of $5 \mu \mathrm{m}$ amplitude, $5 \mathrm{~Hz}$ frequency and position error with $\pm 0.5 \%$ variation in initial values of the parameters $A-D$ is shown in figure 6 .

Performance of the controller in a noisy environment has been observed against varying noise variance (table 3 ). An

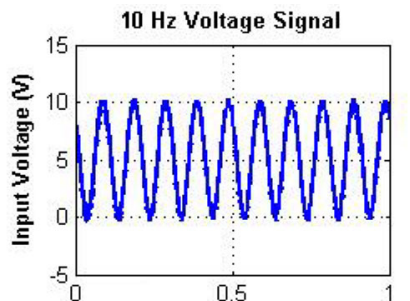

(a) Time (Sec)

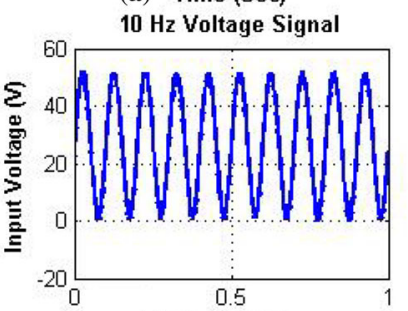

(c) Time (Sec)

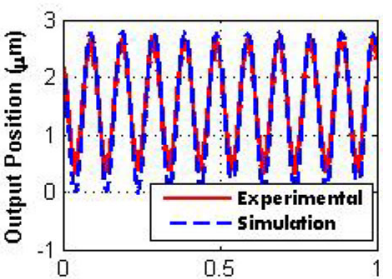

(b) Time(Sec)

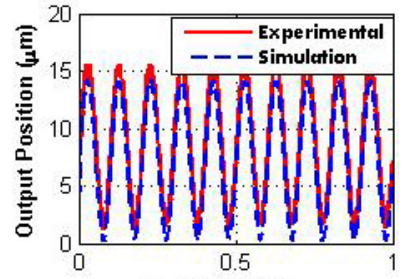

(d) Time(Sec)
Figure 5. (a) $10 \mathrm{~V}, 10 \mathrm{~Hz}$ input voltage of PEA, (b) output position plot of experimental data and simulation model using $10 \mathrm{~V}, 10 \mathrm{~Hz}$ input voltage, (c) $50 \mathrm{~V}, 10 \mathrm{~Hz}$ input voltage of PEA and (d) output position plot of experimental data and simulation model using $50 \mathrm{~V}, 10 \mathrm{~Hz}$ input voltage.

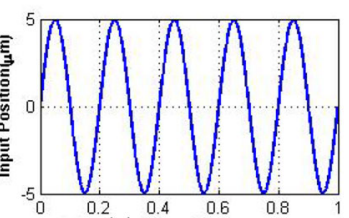

(a) Time (Sec)

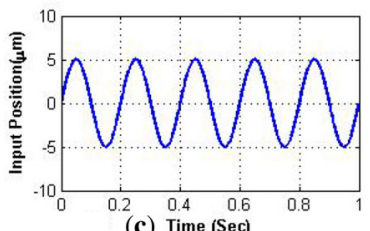

(c) Time (Sec)
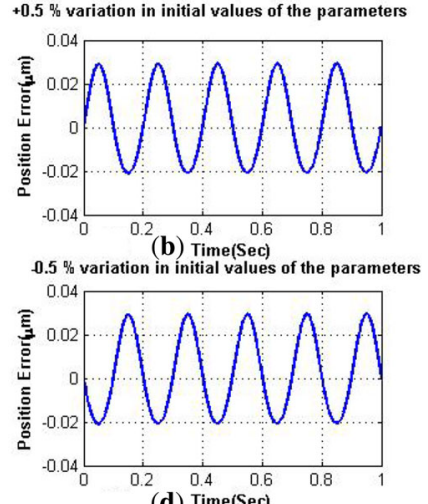

(d) Time(Sec)
Figure 6. (a) $5 \mu \mathrm{m}, 5 \mathrm{~Hz}$ sinusoidal desired input position trajectory, (b) error between output and input position with $+0.5 \%$ variation in initial values of model parameters, (c) $5 \mu \mathrm{m}$, $5 \mathrm{~Hz}$ sinusoidal desired input position trajectory and (d) error between output and input position with $0.5 \%$ variation in initial values of model parameters. 
RMS error of $2.67 \times 10 \mathrm{e}-9$ is observed to a sinusoidal input position of $10 \mu \mathrm{m}, 10 \mathrm{~Hz}$ in a noiseless situation. Normally distributed Gaussian noise has been used to model the noise in simulation. It has been observed that the proposed controller gives satisfactory performance till a noise variance of $10 \mathrm{e}-18$.

The FF controller is implemented using the DsPIC30F4011 microcontroller. The controller generates the required control voltage for a given desired distance. The control voltage is amplified by an amplifier and applied to the PEA. The experimental set-up is shown in figures 7 and 8 . The position error (desired distance - measured distance) is plotted in figure 9a for two different measured data sets. The simulation results of the position error with same desired distance with $\pm 0.1 \%$ variation in initial values of the parameters are shown in figure $9 b$.

Table 3. System performance in noisy environment.

\begin{tabular}{lc}
\hline Gaussian noise variance & RMS error \\
\hline $10 \mathrm{e}-25$ & $2.676 \times 10 \mathrm{e}-9$ \\
$10 \mathrm{e}-20$ & $4.117 \times 10 \mathrm{e}-9$ \\
$10 \mathrm{e}-18$ & $4.117 \times 10 \mathrm{e}-9$ \\
$10 \mathrm{e}-16$ & $3.148 \times 10 \mathrm{e}-8$ \\
$10 \mathrm{e}-15$ & $9.927 \times 10 \mathrm{e}-8$ \\
$10 \mathrm{e}-12$ & $3.138 \times 10 \mathrm{e}-6$ \\
$10 \mathrm{e}-10$ & $3.148 \times 10 \mathrm{e}-5$ \\
$10 \mathrm{e}-09$ & $9.924 \times 10 \mathrm{e}-5$ \\
\hline
\end{tabular}

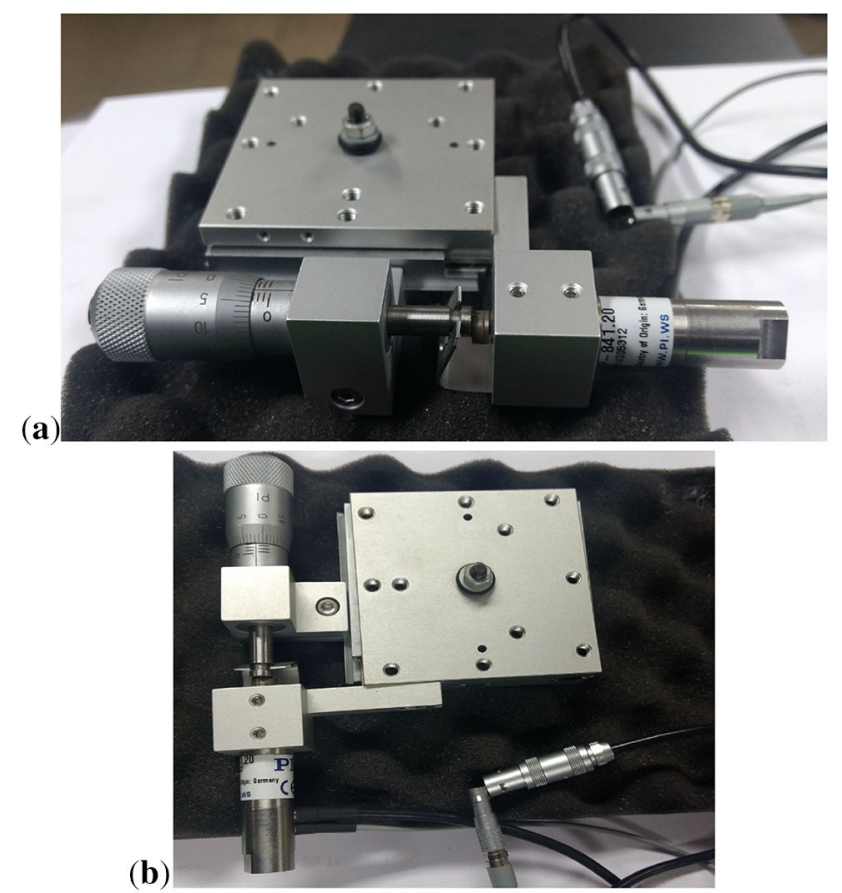

Figure 7. Close-up view of the piezoelectric actuator from Physik Instrumente.

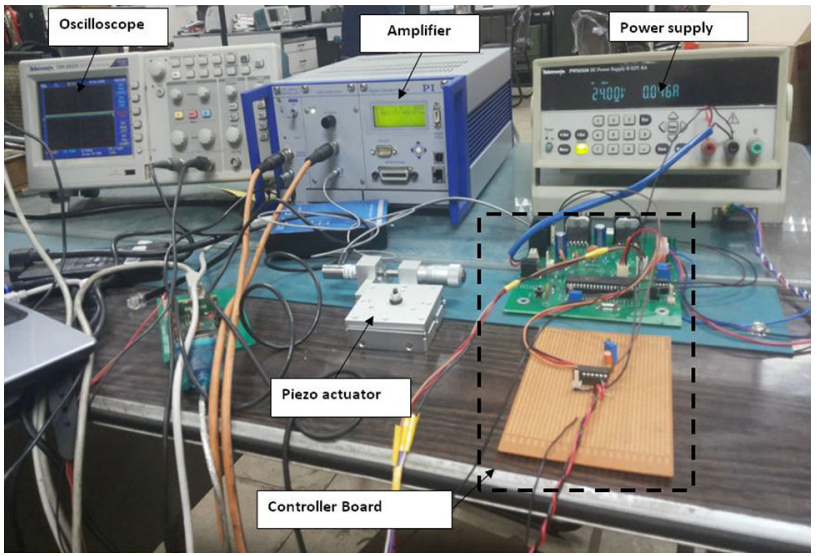

Figure 8. Experimental set-up of feed-forward controller of PEA.

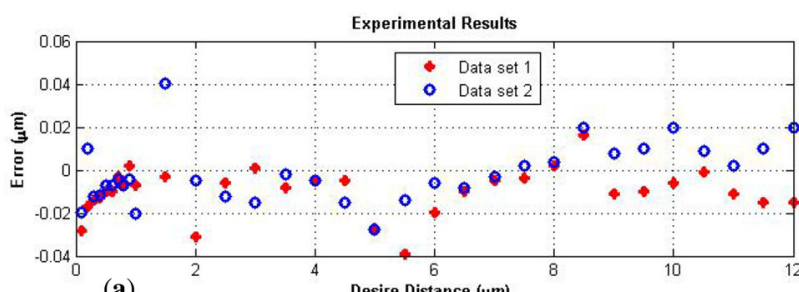

(a)

Simulation Results

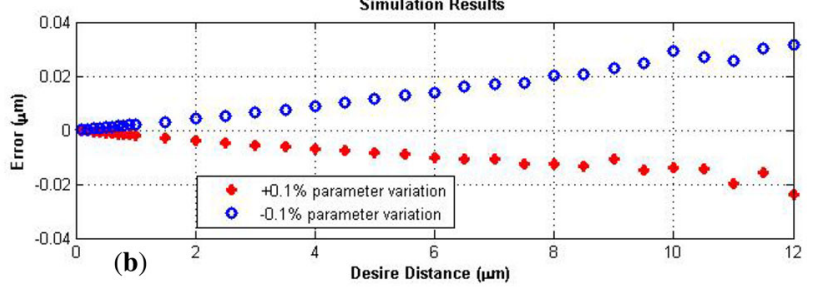

Figure 9. (a) Experimental results of error for different desired distances travelled by PEA. (b) Simulation results of error for different desired distances travelled by PEA with $\pm 0.1 \%$ variation in initial values of the parameters.

\section{Conclusion}

Achieving precise micro/nano-positioning using PEAs is an approach suitable in practical applications for nonlinear system identification, including hysteresis and its control. The identification topology has been based on discrete-time Dahl-based hysteresis modelling of the piezo-actuator with its parameters being identified by an ARX model, which has been validated both in simulation and experiments. A new RLSE technique has been used to control the positioning in FF mechanism and update plant parameters dynamically. The composite control scheme has been simulated in Matlab platform. An RMS error of $0.026 \%$ is obtained to an input sinusoidal tracking signal of $10 \mu \mathrm{m}$, $10 \mathrm{~Hz}$, which is quite useful for several practical applications where accurate positioning is needed like in the field of optics, photonics, measuring technology, nano- 
metrology, micro-lithography, scanning microscopy, etc. Results show impressive performance of the controller against parametric uncertainties as well as in disturbance rejection in the presence of noisy environment. The proposed controller has also been implemented on a real PEA using DsPIC30F4011 microcontroller and experimental results demonstrate the efficacy of the controller.

\section{References}

[1] Schitter G and Stemmer A 2004 Identification and open-loop tracking control of a piezoelectric tube scanner for highspeed scanning-probe microscopy. IEEE Trans. Control Syst. Technol. 12(3): 449-454

[2] Gu G Y and Zhu L M 2013 Motion control of piezoceramic actuators with creep, hysteresis and vibration compensation. Sens. Actuators A: Phys. 197: 76-87

[3] Xu Q 2013 Enhanced discrete-time sliding mode strategy with application to piezoelectric actuator control. IET Control Theory Appl. 7(18): 2153-2163

[4] Gu G Y, Zhu L M and Chun Y S 2014 Modeling and compensation of asymmetric hysteresis nonlinearity for piezoceramic actuators with a modified Prandtl-Ishlinskii model. IEEE Trans. Ind. Electron. 61(3): 1583-1595

[5] Zheng J C and Fu M Y 2013 Saturation control of a piezoelectric actuator for fast settling-time performance. IEEE Trans. Control Syst. Technol. 21(1): 220-8

[6] Zhou M L, Zhang Q and Wang J Y 2014 Feedforwardfeedback hybrid control for magnetic shape memory alloy actuators based on the Krasnosel'skii-Pokrovskii model. PloS One 9: e97086

[7] Rosario T and Ivan I A 2014 Robust structured controllers for piezoelectric microactuators. ISA Trans. 53(6): 1857-1864

[8] Zhou M L, He S B, Hu B and Zhang Q 2015 Modified KP model for hysteresis of magnetic shape memory alloy actuator. IETE Tech. Rev. 32(1): 29-36

[9] Wang X, Alici G and Tan X 2014 Modeling and inverse feedforward control for conducting polymer actuators with hysteresis. Smart Mater. Struct. 23(2): 25015-25023

[10] Janaideh M A and Krejci P 2013 Inverse rate-dependent Prandtl-Ishlinskii model for feedforward compensation of hysteresis in a piezomicropositioning actuator. IEEE/ASME Trans. Mechatron. 18(5): 1498-1507

[11] Zhang J, Merced E, Sepulveda N and Tan X 2014 Modeling and inverse compensation of nonmonotonic hysteresis in $\mathrm{VO}_{2}$-coated microactuators. IEEE/ASME Trans. Mechatron. 19(2): 579-588

[12] Chi Z Q, Jia M P and Xu Q S 2014 Fuzzy PID feedback control of piezoelectric actuator with feedforward compensation. Math. Probl. Eng. 2014: 107184

[13] Xu Q S 2014 Digital sliding-mode control of piezoelectric micro-positioning system based on input-output model. IEEE Trans. Ind. Electron. 61(10): 5517-5526

[14] Al-Wahab M A, Kasper R, Kostadinov K, Chakarov D and Tiankov T 2008 Structured piezo-ceramic mechatronic handling devices for micro and nano manipulations. In: Proceedings of the 5th International Symposium on
Mechatronics and Its Applications (ISM08), Amman, 27-29 May, pp. 1-6

[15] Al Mamun A and Yao K 2015 Analysis and modeling of hysteresis of piezoelectric micro-actuator used in high precision dual-stage servo system. Control Theory Technol. 13(2): 184-203

[16] Viswamurthy S R and Ganguli R 2007 Modeling and compensation of piezoceramic actuator hysteresis for helicopter vibration control. Sens. Actuators A: Phys. 135(2): 801-810

[17] Viswamurthy S R, Rao A K and Ganguli R 2007 Dynamic hysteresis of piezoceramic stack actuators used in helicopter vibration control: experiments and simulations. Smart Mater. Struct. 16(4): 1109

[18] Liaw H C and Shirinzadeh B 2011 Robust adaptive constrained motion tracking control of piezo-actuated flexurebased mechanisms for micro/nano manipulation. IEEE Trans. Ind. Electron. 58(4): 1406-1415

[19] Rakotondrabe M and Ivan I A 2011 Development and force/position control of a new hybrid thermo-piezoelectric micro gripper dedicated to micromanipulation tasks. IEEE Trans. Autom. Sci. Eng. 8(4): 824-834

[20] Leaning K K and Devasia S 2007 Feedback linearized inverse feedforward for creep, hysteresis and vibration compensation in AFM piezoactuators. IEEE Trans. Control Syst. Technol. 15(5): 927-935

[21] Devasia S, Eleftheriou E and Moheimani S 2007 A survey of control issues in nanopositioning. IEEE Trans. Control Syst. Technol. 15(5): 802-823

[22] Li Y and Xu Q 2010 Adaptive sliding mode control with perturbation estimation and PID sliding surface for motion tracking of a piezo-driven micromanipulator. IEEE Trans. Control Syst. Technol. 18(4): 798-810

[23] Chen X 2011 High precision adaptive control for XYtable driven by piezo-actuator. In: Proceedings of the 2011 International Conference on Advanced Mechatronic Systems, Zhengzhou, China, August 11-13

[24] Liu D and Fujii F 2014 An adaptive internal model control system of a piezo-ceramic actuator with two RBF neural networks. In: Proceedings of the 2014 IEEE International Conference on Mechatronics and Automation, August 3-6, Tianjin, China

[25] Lin F J, Shieh H J, Huang P K and Teng L T 2006 Adaptive control with hysteresis estimation and compensation using RFNN for piezo-actuator. IEEE Trans. Ultrason. Ferroelectr. Freq. Control 53(9): 1649-1661

[26] Astrom K J and Wittenmark B 1994 Adaptive control, 2nd ed. Mineola, N.Y.: Dover Publications Inc.

[27] Li J and Yang L 2014 Adaptive PI-based sliding mode control for nanopositioning of piezoelectric actuators. Math. Probl. Eng. vol. 2014, Article ID 357864, pp. 10, DOI: $10.1155 / 2014 / 357864$

[28] Shieh H J and Hsu C H 2008 An adaptive approximatorbased backstepping control approach for piezoactuator-driven stages. IEEE Trans. Ind. Electron. 55(4): 1729-1738

[29] Zhang L J, Yang L X, Zhang X W and Sun L N 2010 Adaptive output feedback control with feedback feedfoward compensator for piezoactuator-driven stages. In: Proceedings of the 8th World Congress on Intelligent Control and Automation, July 6-9, Jinan, China

[30] Tao G and Kokotovic P 1995 Adaptive control of plants with unknown hysteresis. IEEE Trans. Autom. Control 40(2): 200-212 
[31] Zhou M, Yang P, Wang J and Gao W 2016 Adaptive sliding mode control based on Duhem model for piezoelectric actuators. IETE Tech. Rev., DOI: 10.1080/ 02564602.2015.1126202

[32] Minh T V, Linh N M and Chen X 2016 Tracking control of piezoelectric actuator using adaptive model. Robot. Biomim. 3: 5, DOI: 10.1186/s40638-016-0039-x

[33] Adriaens H J M T A, Koning W L D and Banning R 2000 Modeling of piezoelectric actuators. IEEE/ASME Trans. Mechatron. 5(4): 331-334

[34] Jang M J, Chen C L and Lee J R 2005 Modeling and control of a piezoelectric actuator driven system with asymmetric hysteresis. J. Franklin Inst. 346(1): 17-32

[35] Zhang Y L, Han M L, Yu M Y, Shee C Y and Ang W T 2012 Automatic hysteresis modeling of piezoelectric micromanipulator in vision-guided micromanipulation systems. IEEE/ ASME Trans. Mechatron. 17(3): 547-553

[36] Song G, Zhao J, Zhou X and De Abreu-Garcia J 2005 Tracking control of a piezoceramic actuator with hysteresis compensation using inverse Preisach model. IEEE/ASME Trans. Mechatron. 10(2): 198-209
[37] Rakotondrabe M 2011 Bouc-Wen modeling and inverse multiplicative structure to compensate hysteresis nonlinearity in piezoelectric actuators. IEEE Trans. Autom. Sci. Eng. 8(2): $428-431$

[38] Xu Q and Li Y 2010 Dahl model-based hysteresis compensation and precise positioning control of an XY parallel micromanipulator with piezoelectric actuation. J. Dyn. Syst. Meas. Control 132: 041011.

[39] Deng L and Tan Y 2008 Modeling of rate-dependent hysteresis in piezoelectric actuators. In: Proceedings of the IEEE International Conference on Control Applications, CCA 2008, DOI: 10.1109/CCA.2008.4629578

[40] Liu L, Tan K, Chen S, Teo T and Lee T 2012 Identification and compensation of hysteretic dynamics of piezoelectric actuators for accurate and fast scanning. In: Proceedings of the IEEE Conference on Industrial Electronics and Applications (ICIEA), DOI: 10.1109/ICIEA.2012.6360987

[41] Lampaert V and Sweavers J 2001 Online identification of hysteresis functions with non-local memory. In: Proceedings of the 2001 IEEE/ASME International Conference on Advanced Intelligent Mechatronics, DOI: 10.1109/AIM.2001.936774 